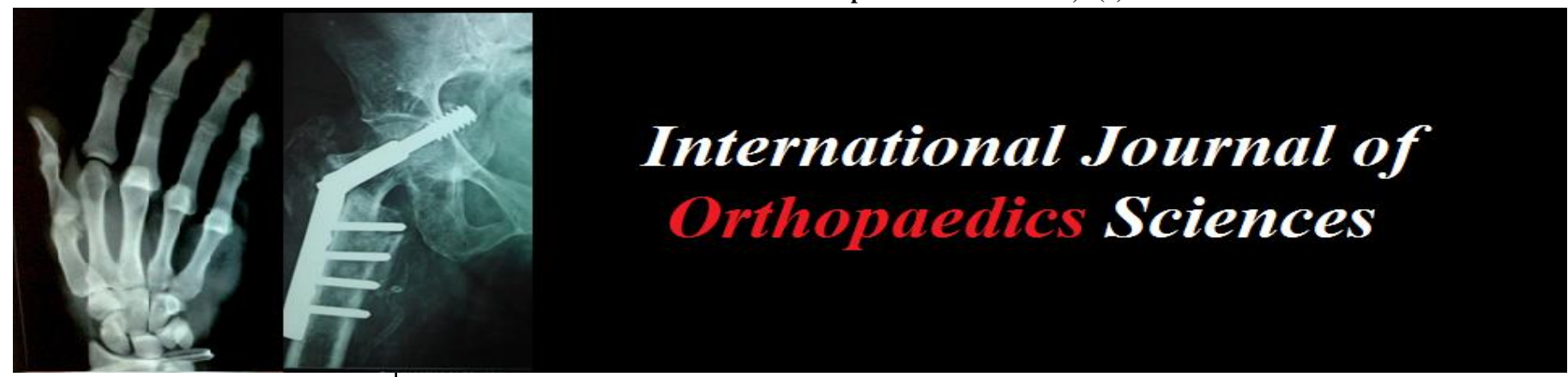

E-ISSN: 2395-1958

P-ISSN: 2706-6630

IJOS 2019; 5(4): 24-28

(C) 2019 IJOS

www.orthopaper.com

Received: 14-08-2019

Accepted: 18-09-2019

Dr. Santosh Kumar Sahu Assistant Professor, Department of Orthopaedics, IMS \& SUM Hospital, Bhubaneswar, Odisha, India

Dr. Arindam Chatterjee PGT of Orthopaedics at IMS \& SUM Hospital, Bhubaneswar, Odisha, India
Corresponding Author: Dr. Santosh Kumar Sahu Assistant Professor, Department of Orthopaedics, IMS \& SUM Hospital, Bhubaneswar, Odisha, India

\section{Hemiarthroplasty versus PHILOS for the management of Neer group (IV-VI) Proximal humeral fractures: A retrospective study}

\section{Dr. Santosh Kumar Sahu and Dr. Arindam Chatterjee}

DOI: https://doi.org/10.22271/ortho.2019.v5.i4a.1641

Abstract

Neer Group (IV-VI) Proximal humeral fractures often are related to persistent disability despite surgical treatment. The optimal surgical management of theses fractures in elderly patients remains unclear. We retrospectively compared the outcome after open reduction and internal fixation with the PHILOS and primary hemiarthroplasty in patients with Neer Group IV-VI fractures focusing on Speed of recovery, shoulder function, health-related quality of life, and complications.

Keywords: Hemiarthroplasty, proximal humerus fractures, PHILOS, Neer classification

\section{Introduction}

Fracture of the proximal humerus is a common injury, especially in elderly patients with osteoporotic bone. Neer introduced a fracture classification, which is still widely used ${ }^{[13]}$. In its Group (IV-VI) he gathered three and four-part fractures and dislocations. Displaced fractures of the articular surface (impression or split) also are included in this group, because the non-crushed part of the humeral head is extruded from the joint during impact. Neer recommended open reduction and internal fixation (ORIF) for three-part fracture dislocations and primary hemiarthroplasty (HA) for four-part fracture dislocations and for fractures with greater than $50 \%$ of cartilage-covered articular defect. PHILOS (Proximal Humerus Interlocking Osteosynthesis) have been developed for fractures of the proximal humerus with these implants, better biomechanical stability could be achieved. Anatomic reconstruction for severely displaced fractures and fractures with glenohumeral dislocations aiming to achieve superior function compared with primary Hemi-Arthroplasty has been reported with PHILOS. While for undisplaced fractures, literature strongly suggests non operative treatment ${ }^{[1-3]}$, the treatment of displaced fractures is still controversial and challenging. With continued advancement of techniques and implants such as locking plates, surgical fixation of proximal humerus fractures has been increasing in popularity. However, the reported complication rates in humeral head preserving procedures continue to be high. In particular, the rate of osteonecrosis remains unchanged even with the most modern of techniques. It is clear that the prevalence of osteonecrosis after proximal humerus fractures increases over time ${ }^{[3]}$. Fractures with a humeral head split and complex three- and four-part fractures are also at risk for the development of malunion and osteonecrosis after internal fixation ${ }^{[5,6]}$. Both shoulder hemiarthroplasty and, more recently, reverse total shoulder arthroplasty (RTSA) are indicated for these more complex fractures with high complication rates with humeral head preserving procedures. Joint replacement has limited indications, strict selection of patients, and significant invasivity, but faster active recovery ${ }^{[4,5]}$. The use of hemiarthroplasty for treating displaced three or four-part fractures was initially reported by Neer [12]. Multiple studies subsequently reported inconsistent results for ROM and function [2-4, 7-11]. Complications include displacement of the tuberosity fragments, persistent pain, glenohumeral joint space narrowing, and heterotrophic ossification. 


\section{Objectives}

The objectives of the present study was to compare the PHILOS with primary hemiarthroplasty for treatment of specific Neer Group (IV-VI) fractures and assess the functional outcome in patients admitted to Orthopaedic Department of IMS\&SUM Hospital, Bhubaneswar from $1^{\text {st }}$ January 2016 to $31^{\text {st }}$ December 2018 in terms of pain, shoulder stability and range of movements at shoulder joint Speed of recovery, shoulder function, health-related quality of life, and complications.

\section{Methodology}

It was a retrospective study conducted at IMS\&SUM Hospital, Bhubaneswar during the period of January 2016 to December 2018.A total of 60 patients more than 60 years age, with a Neer Group (IV-VI) fracture of the Proximal Humerus were treated in IMS \& SUM Hospital, Bhubaneswar. In 21 (35\%) patients, primary HA was performed, PHILOS was performed in $39(65 \%)$ patients. Both groups were similar in allcriteria. All patients with un-displaced proximal humerus fractures, pathological fractures and those with compound fractures with or without neurovascular deficit, fractures older than 21 days, multiple co-morbidities, polytrauma, unwilling patients were excluded from our study. We analyzed all initial AP, Lateral \& Axillary Radiographs and available CT scans. We reviewed these patients charts retrospectively. Patients preoperative history and medications were documented. AP and LAT radiographic examinations at the time of injury, after surgery, and at the time of last follow-up 12 months were available for all patients.
Functional and clinical assessments using the age and gender specific Constant-Murley Score (CMS) and the Visual Analogue Scale were performed at the last followup or at the time of a complication. No apparent differences between the two groups regarding group size, age, sex, or comorbidities were seen. At minimum followup of 3,6,9,12 months, Constant-Murley score, were calculated. Results with complications were analysed in both the groups and there were no significant differences between the two groups as regards Constant-Murley scores.

\section{Surgical Procedure}

Patients were randomized to either Hemiarthroplasty or PHILOS group. PHILOS was performed in 39 cases with the patient in supine or in beach chair position on a radiolucent table and a deltopectoral approach was used. The fracture was reduced and provisionally stabilized with Kirschner wires. The reduction was confirmed as adequate with use of image intensification. The PHILOS was positioned, at least 5-8 $\mathrm{mm}$ distally to the upper end of the greater tuberosity and $2 \mathrm{~mm}$ posteriorly to the bicipital groove. Care was taken to ensure that a sufficient gap is maintained between the plate and the tendon of the long head of the biceps. When fracture reduction and subsequent screw positioning was considered adequate, the plate was fixed definitively with the insertion of angular stable screws in the humeral head. The use of angular stable or standard cortical screws for the humeral shaft holes was left to the treating surgeon. A final image intensifier cheverify.
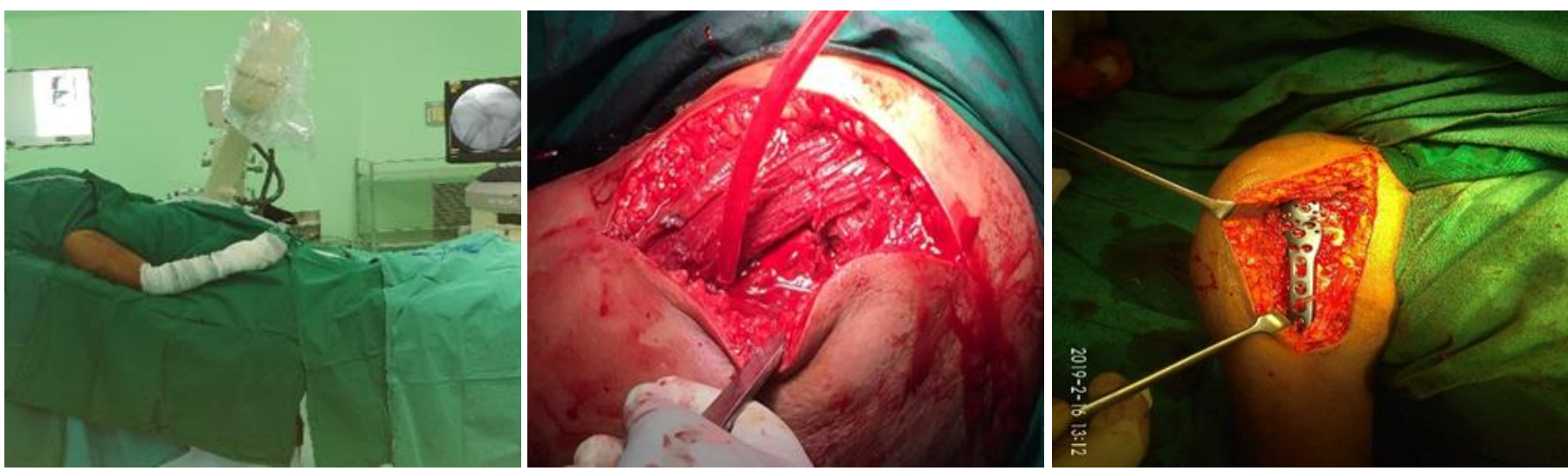

Fig 1: (PHILOS)

For the hemiarthroplasty group, which included 21 patients, a modular prosthesis (Depuy Global Fx System) was used. Its stem was 130-mm long and $8 \mathrm{~mm}$ thick for 9 patients; and 12 received stem size of $140 \mathrm{~mm}$ long and $10 \mathrm{~mm}$ thick size. Its humeral head thickness was one size less or equal to the extracted humeral head and included $46 \times 18 \mathrm{~mm}, 46 \times 15 \mathrm{~mm}$, $40 \times 18 \mathrm{~mm}$, and $40 \times 15 \mathrm{~mm}$. Patients were placed in the a semi-Fowler beach chair position. For all cases, deltopectoral approach was used. The short head of biceps and the coracobrachialis muscle were displaced medially before access can be gained to the anterior aspect of shoulder joint. With the greater and lesser tuberosities retracted out of the way by sutures, a bone clamp was used to retrieve the fractured humeral head. We measured the resected humeral head for height and diameter using the Humeral Head
Template. The selected humeral head component must be approximate the resected humeral head height and radius of curvature. The Global Advantage Humeral Head trials for the fracture set range from 15, 18 and $21 \mathrm{~mm}$ heights and 44, 48 and $52 \mathrm{~mm}$ diameters are available. After selection of the humeral head component we extracted cancellous graft from the head for secure tuberosity fixation. Medullary canal was preparedby serial reaming $(6-12 \mathrm{~mm})$. We attached the proper size trial head to a trial stem, and placed it into the intramedullary canal, with elbow in 90-degree flexion and parallel to the floor and in zero degree rotation while maintaining 30-degree retroversion. Appropriate size head was then fixed over the stem. Greater and lesser tuberosities were reconstructed over the implant withthe help of nonabsorbable sutures. 

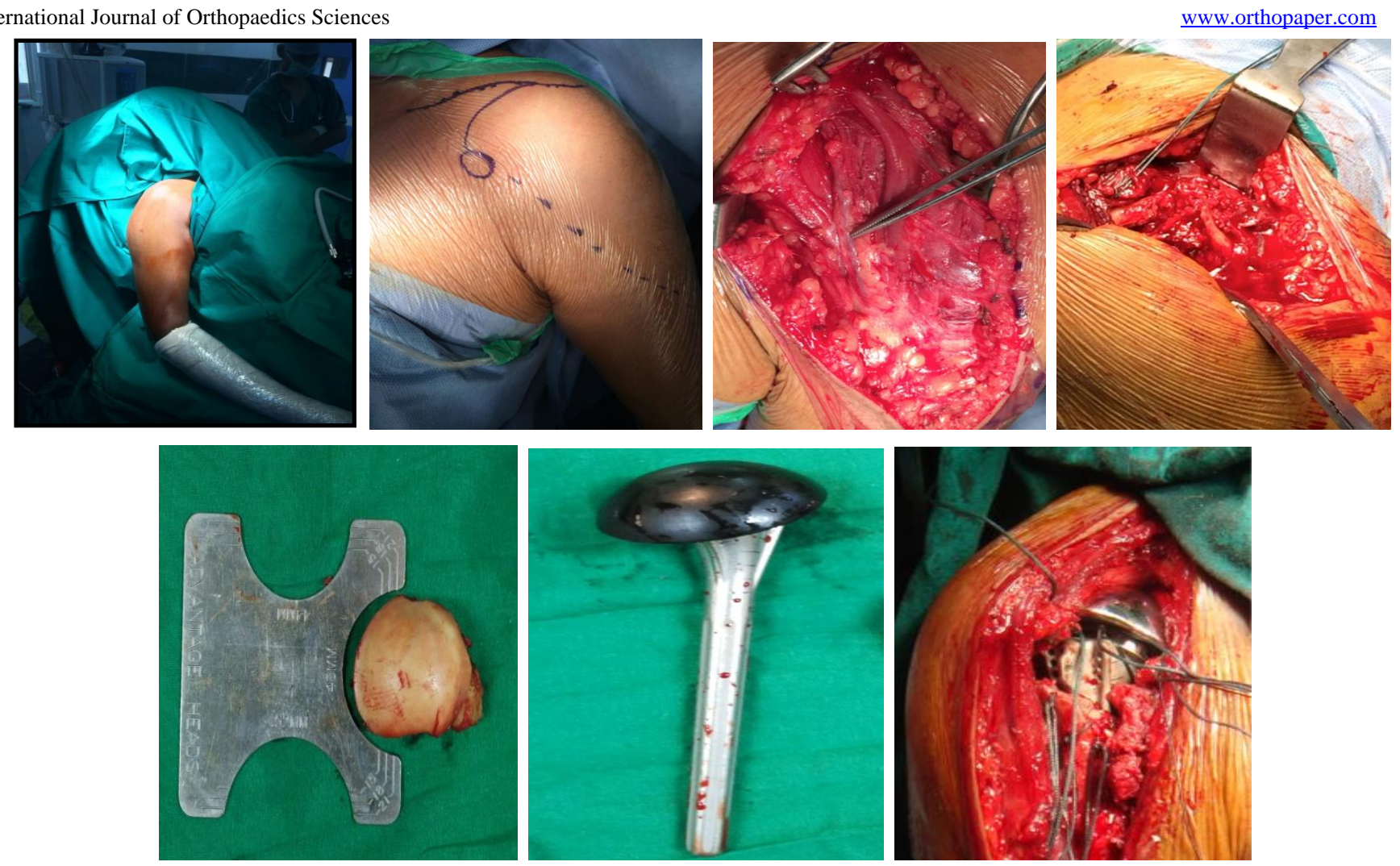

Fig 2: (Hemiarthroplasty)

All the surgical procedures were performed by the same qualified surgeon. Antibiotic prophylaxis with first generation Cephalosporins are given intravenously, preoperatively and post operatively for 48hours.Postoperatively the patients received a thromboprophylaxis during the stay (e.g. LMW Heparin or equivalent).In terms of rehabilitation standard protocols were followed. Arm slings are given for the first 6 weeks combined with mobilization instructions. 2 weeks postoperatively, active range of motion were given, after another 2 weeks active external rotation was encouraged and initiated. Rehabilitation was supervised by a Physiotherapist of the hospital.

Table 1: (Constant Murley Score: in all the patients)

\begin{tabular}{|c|c|c|}
\hline CMS Score & Hemi Arthroplasty (21) & PHILOS (39) \\
\hline Poor & 2 & 1 \\
\hline Moderate & 4 & 8 \\
\hline Good & 8 & 12 \\
\hline Excellent & 7 & 18 \\
\hline
\end{tabular}

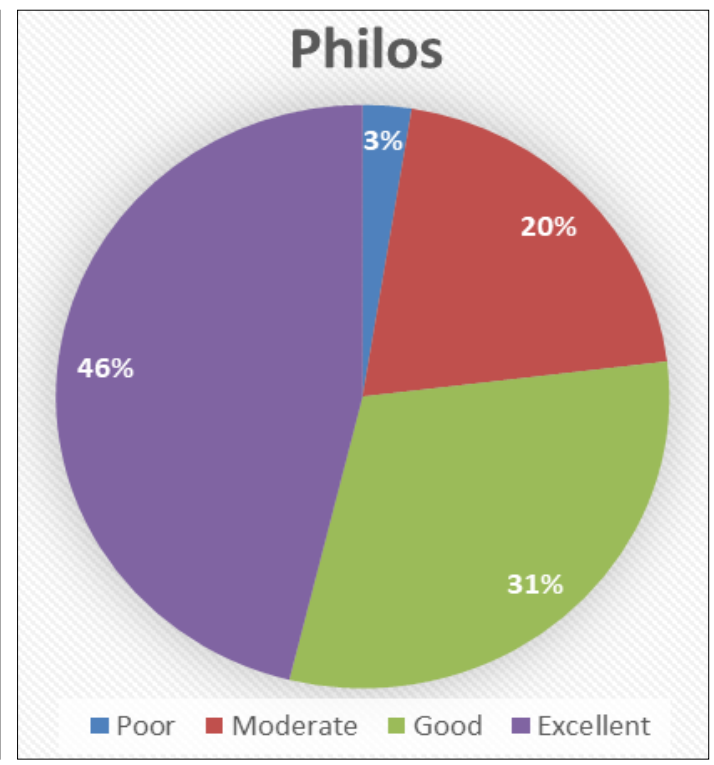

Fig 3: (Constant Murley Scoring System) 
Table 2: (Shoulder Range of Movement)

\begin{tabular}{|c|c|c|}
\hline Shoulder Range of Movement & Hemiarthroplasty & PHILOS \\
\hline Good & 12 & 30 \\
\hline Moderate & 7 & 8 \\
\hline Poor & 2 & 1 \\
\hline
\end{tabular}

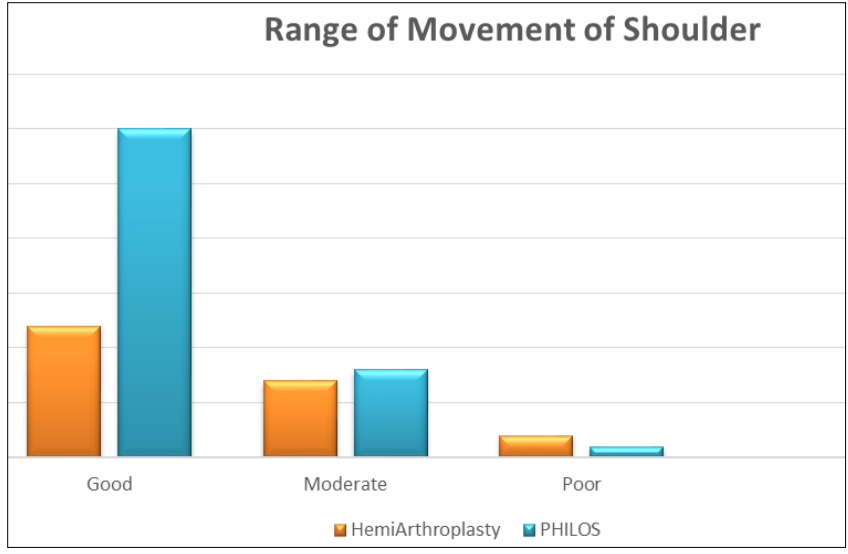

Fig 4: (ROM of shoulder)

\section{Results}

Clinical and radiographic complication rates were comparable in both groups at the last followup. The complication rates were $30.8 \%$ (12 of 39) for the PHILOS group and 33.33\% (7 of 21) for the HA group. The most frequent complication in the PHILOS group was stiffness of the shoulder joint in (11 of $39 ; 28.2 \%$ ) who later improved by physiotherapy. Screw cutout attributable to secondary collapse of the fracture was not seen. AVN of the head was seen in $(1$ of $39 ; 2.6 \%)$ case that later was managed by Hemi-Arthroplasty. In the HA group, posterior malreduction of the greater tuberosity was seen (1 of $21 ; 4.8 \%)$ case that healed in this position, 3 of $21(14.3 \%)$ had an acromio-humeral distance less than $7 \mathrm{~mm}$ at the last radiographic follow up. Of those, 1 patient had complete resorption of the greater tuberosity with primary posterior malposition and 2 with primary correct reduction. No sign of prosthetic loosening was seen in any patient at the time of the last radiographic followup. Post-operative stiffness of shoulder in HA group was seen in 7 of $21(33.3 \%)$ out of which 5 patients $(71.42 \%$ of affected patients) have shown improvement with physiotherapy.

The findings in the Constant-Murley scores between the HA and PHILO Streated fractures revealed Excellent outcomes in $33 \%$ in HA, $46 \%$ in PHILOS, Good outcomes in $38 \%$ in HA, $31 \%$ in PHILOS, Moderate in $19 \%$ in HA, $20 \%$ in PHILOS, Poor outcomes in (10\% in HA, 3\% in PHILOS).In patients operated with PHILOS, Range of Movement of Shoulder post operatively was Excellent in (30 of 39; 76.9\%), Moderate in (8 of 39; 20.5\%), Poor in (1 of 39; 2.6\%). In patients operated with HA, range of movement of shoulder post operatively was Excellent in (12 of $21 ; 57.1 \%)$, Moderate in (7 of $21 ; 33.3 \%)$, Poor in (2 of $21 ; 9.6 \%)$.

Table 3: (Patient Characteristics)

\begin{tabular}{|c|c|c|}
\hline Patient Characteristics & Hemi Arthroplasty (21) & PHILOS (39) \\
\hline Male / Female & $9 / 12$ & $16 / 23$ \\
\hline Side Affected (L/R) & $7 / 14$ & $14 / 25$ \\
\hline Delay to Surgery (in days) & $4.95^{*}$ & $3.02^{*}$ \\
\hline Mean age range in years & $66.3(60$ to 81$)$ & $63.6(61$ to 78$)$ \\
\hline
\end{tabular}

*mean

\section{Conclusion}

It can be concluded from the above study that though the functional outcomes and quality of life in ORIF group in comparison to HA group was better, although most outcomes were not significantly different. The overall acceptable outcome and limited need for secondary surgical interventions in the ORIF group indicate that ORIF is a sufficient method for elderly patients with lower functional demands.

\section{References}

1. Green A, Norris T. Proximal humerus fractures and fracture dislocations. In: Browner B, Jupiter J, Levine A, Trafton P, editors. Skeletal trauma: basic science, management and reconstruction. 3. Philadelphia: Saunders, 2003, pp. 1532-1624.

2. Court-Brown CM, Garg A, Mc-Queen MM. The epidemiology of proximal humeral fractures. Acta Orthop Scand. 2001; 72(4):365-371. DOI: $10.1080 / 000164701753542023$

3. Harrison AK, Gruson KI, Zmistowski B et al. Intermediate outcomes following percutaneous fixation of proximal humeral fractures. J Bone Joint Surg Am. 2012; 94(13):1223-1228. DOI: 10.2106/JBJS.J.01371

4. Spross C, Platz A, Rufibach K et al. The PHILOS plate for proximal humeral fractures-risk factors for complications at one year. J Trauma Acute CareSurg. 2012; 72(3):783-792. DOI: 97/TA.0b013e31822c1b5b.

5. Connor PM, Flatow EL. Complications of internal fixation of proximal humeral fractures. Instr Course Lect. 1997; 46:25-37.

6. Gerber C, Werner CM, Vienne P. Internal fixation of complex fractures of the proximal humerus. J Bone Joint Surg Br. 2004; 86(6):848-855. DOI: 10.1302/0301620X.86B6.14577.

7. Brorson S, Olsen BS, Frich LH et al. BMC Surgeons agree more on treatment recommendations than on classification of proximal humerus fractures. Musculoskelet Disord. 2012; 13(1):114. DOI: 10.1186/1471-2474-13-114.

8. Zyto K. Non-operative treatment of comminuted fractures of the proximal humerus in elderly patients. Injury. 1998; 29(5):349-352. DOI: 10.1016/S0020-1383(97)00211-8.

9. Neviaser AS, Hettrich CM, Dines JS, Lorich DG. Rate of avascular necrosis following proximal humerus fractures treated with a lateral locking plate and endosteal implant. Arch Orthop Trauma Surg. 2011; 131(12):16171622. DOI: 10.1007/s00402-011-1366-6.

10. Frankle MA, Chacon-Balados A, Cuff D. Reverse shoulder prosthesis for acute and chronic fractures. In: Dines DM, Laurencin CT, Williams GR, editors. Arthritis and Arthroplasty: The Shoulder. Philadelphia: PA, Saunders, 2009, pp. 218-231.

11. Liu J, Li SH, Cai ZD et al. Outcomes, and factors affecting outcomes, following shoulderhemiarthroplasty for proximal humeral fracture repair. J Orthop Sci. 2011; 16(5):565-572. DOI: 10.1007/s00776-011-0113-8.

12. Sirveaux F, Navez G, Roche O, Molé D, Williams MD. Reverse prosthesis for proximal humerus fracture, technique and results. Tech Shoulder Elbow Surg. 2008; 
9:15-22. DOI: 10.1097/BTE.0b013e31815dca3c.

13. Christian Spross MD, Andreas Platz MD, Matthias Erschbamer MD, PhD Thomas Lattmann MD, Michael Dietrich MDSurgical Treatment of Neer Group VI Proximal Humeral Fractures, Retrospective Comparison of PHILOS ${ }^{1}$ and Hemiarthroplasty The Association of Bone and Joint Surgeons, 2011.

14. Sudkamp N, Bayer J, Hepp P, Voigt C, Oestern H, Kaab $\mathrm{M}$ et al. Open reduction and internal fixation of proximal humeral fractures with use of the locking proximal humerus plate: results of a prospective, multi- center, observational study. J Bone Joint Surg Am, 2009.

15. Neer CS 2nd. Displaced proximal humeral fractures: I. Classifi- cation and evaluation. J Bone Joint Surg Am. 1970.4. Handschin AE, Cardell M, Contaldo C, Trentz O, Wanner GA. Functional results of angular-stable plate fixation in displaced proximal humeral fractures. Injury, 2008. 